\title{
RTM processing and electrical performance of carbon nanotube modified epoxy/fibre composites
}

\author{
Elisabete F. Reia da Costa ${ }^{{ }^{*}}$, Alexandros A. Skordos ${ }^{1}$, Ivana K. Partridge ${ }^{1}$, Amir Rezai ${ }^{2}$ \\ ${ }^{1}$ Cranfield University, Composites Centre, MK43 OAL, UK \\ ${ }^{2}$ BAE Systems, Advanced Technology Centre, BS34 7QW, UK \\ *Corresponding author's email: e.f.r.costa@cranfield.ac.uk
}

\begin{abstract}
This investigation focuses on nanoparticle filtration in the processing of multiscale carbon and glass fibre composites via resin transfer moulding. Surface modified and unmodified carbon nanotubes (CNTs) were incorporated into a commercial epoxy resin. The dispersion quality was evaluated using electrical measurements of the liquid suspensions. The manufacturing process was adapted to the challenges posed by the modified rheological behaviour of the CNT loaded resin. Nanoparticle filtration was observed; with some of the unmodified systems following so called 'cake filtration' behaviour. This resulted in non linear flow behaviour that deviated from the ideal response observed in RTM filling in conventional composites. The electrical conductivity of relatively high fibre volume fraction multiscale carbon and glass laminates increased by less than an order of magnitude with the addition of the nanotubes.
\end{abstract}

Keywords: A. Nano-structures; B. Electrical properties; E. Resin transfer moulding; E. Resin Flow 


\section{Introduction}

The high electrical conductivity of carbon nanotubes (CNTs) coupled with their outstanding mechanical and thermal properties and very high aspect ratios make them very attractive polymer matrix fillers. Minimal CNT loadings in the resin can result in considerable improvements in the electrical properties of the produced nanocomposites [1, 2]. The transfer of the unique CNT properties to the polymer nanocomposite relies on the achievement of a homogenous and stable dispersion of carbon nanotubes in the polymer medium [3, 4]. As produced CNTs tend to form aggregates due to their high specific surface area, which can be above $1000 \mathrm{~m}^{2} / \mathrm{g}$ [5]. Both physical and chemical dispersion methods have been used to obtain satisfactory and stable suspensions. Ultrasonication, shear mixing and triple roll milling are among the most common physical dispersion methods, whilst CNT surface functionalisation, polymer wrapping and surfactant assistance promote the bonding of nanotubes to the polymer [6]. For thermosetting resins the dispersion takes place in the prepolymer state, followed by temperature activated cure. During cure, CNT reaggregation is likely to occur, disrupting the previous state of dispersion $[7,8]$.

In recent years fibrous nanoparticles have been introduced into the manufacture of high performance continuous fibre reinforced structural composites for aerospace applications, mainly in an attempt to improve their out-of-plane mechanical properties [9]. This has focused research on the production of these types of hierarchically structured materials, usually referred to as 'multiscale polymer composites'. These combine carbon nanotubes or nanofibres with conventional fibre reinforcements by employing and adapting existing liquid moulding processes such as Resin Transfer Moulding (RTM) [10, 11] and Vacuum Assisted Resin Transfer Moulding (VARTM) [12-15]. The incorporation of CNTs has been achieved either by direct dispersion of the CNTs in the liquid polymer matrix $[16,17]$ or by an attachment of CNTs onto the fibre reinforcement $[9,18,19]$. The first approach is advantageous due to its simplicity and compatibility with the conventional industrial practice, but is limited to CNT content below 0.3 
wt $\%$ as stable and homogenous dispersions of suspensions containing higher CNT loadings may lead to unacceptably high resin viscosities [20].

In addition to the viscosity issues related to high CNT contents, filtration of the nanofiller by the fibrous medium may also lead to inadequate final component quality. The so called 'deep bed filtration' and 'cake filtration' are the main filtration mechanisms which can take place during liquid moulding of composites with filled resins [21-23]. Cake filtration takes place when the particle size is larger than the available pore size. This results in the deposition of particles, the 'filter cake', which can build-up ahead of the fibrous medium as soon as the suspension enters the mould. That situation refers to a 'macroscopic' cake filtration. Alternatively, 'microscopic' cake filtration is likely to occur along the reinforcement length, due to its dual scale nature of inter and intra tow channels. 'Microscopic' cake filtration can occur especially during tow impregnation, when the suspension is characterised by CNT aggregates larger than the inter tow pore dimension. Deep bed filtration is characterised by the capture of particles dimensionally smaller than the pore channels. Continuous capture of particles through deep bed filtration can result in the reduction of the available flow channels dimensions leading ultimately to cake filtration at a microscopic level. Both mechanisms cause clogging of the fibrous porous media, slowing down the resin flow front progression and thus resulting in longer infusion cycles and filler concentration gradients. Despite the problems associated with the processing of such multiscale composite systems, improvements both in mechanical and electrical conductivity properties have been reported $[9,20]$.

This research focuses on the transfer of the enhanced electrical properties of the carbon nanomodified resin to the final composite structure. Adequate transfer of these properties is essential for the manufacture of multifunctional composite structures. An adequate selection of particle loading and dispersion technique is essential for guaranteeing the processability of these materials and ultimately the transferability of their properties. However, particle filtration by the fibrous porous media is likely to occur, and if its mechanisms are not understood this transfer can be compromised. This urges the need for a modelling tool able to describe the process and predict particle content in the final composite. 
In the present work two grades of unmodified and one grade of surface modified CNTs are used to alter the properties of an RTM-grade epoxy matrix. Microscopy is utilised to evaluate the state of dispersion in the liquid and in the solid states whilst electrical conductivity measurements on the liquid suspensions allow the selection of the most appropriate dispersion methods. These suspensions are used to infuse both carbon and glass fibre fabrics using the RTM process, highlighting certain adjustments that need to be made to the processing conditions. The extent and nature of nanoparticle filtration are identified microscopically and macroscopically and the electrical conductivity of the produced composites is evaluated. 


\section{Materials and Methods}

\subsection{Materials}

The two-component low viscosity thermoset resin system used in this work comprised Huntsman Araldite LY564 epoxy resin with Huntsman Aradur 2954 amine hardener, in weight ratio of 100/35. Commercial multiwalled carbon nanotubes (MWCNTs) were used to modify the resin. Fig. 1 shows as-produced dry MWCNT nanoparticles P940 and C100, supplied by Thomas Swan and CNT Co. respectively. P940 MWCNTs are manufactured by a CVD process; their average diameter is in the range of $10-12 \mathrm{~nm}$ and their length is in the order of tens of microns. C100 MWCNTs manufactured by CVD have 10-40 nm average diameter and are 1 to $25 \mu \mathrm{m}$ long. BAE Systems proprietary surface modified MWCNTs were supplied in a prebatch form. The same CNT content is reported for both the liquid and cured resins, however, this value corresponds to the cured state concentration of CNTs, which takes into account the amount of hardener added after the dispersion process.

The reference materials, either the liquid or the cured resin, and the fibrous composite are referred to as 'control'. Pseudo unidirectional uniweave carbon fabric $\left(280 \mathrm{~g} / \mathrm{m}^{2}-\mathrm{Hexcel}\right)$ and a uniweave glass fabric ( $480 \mathrm{~g} / \mathrm{m}^{2}-\mathrm{SP}$ Gurit) were utilised as reinforcements.

\subsection{Dispersion processing}

A process step of dispersing the nanoparticles in the resin was performed prior to the injection of the nanofilled resin into the mould. The suspensions containing unmodified nanotubes (C100 and P940) were prepared in-house, while the chemically modified CNTs were supplied as a ready to use epoxy suspension. C100 nanotubes were initially dispersed in the epoxy prepolymer at a loading of $1.5 \mathrm{wt} \%$ by four passes at $50 \mathrm{rpm}$ through a triple roll mill (TRM Torrey Hills Technologies). This high concentration suspension was then diluted to a concentration of $0.3375 \mathrm{wt} \%$, which upon addition of the hardener results in the desired final 
$0.25 \mathrm{wt} \%$ loading. The suspension was shear mixed for 15000 cycles in a DISPERMAT CN F2 high shear mixer and then ultrasonicated using a Branson S-450D horn with a total energy input of $650 \mathrm{~J} / \mathrm{g}$. Batches of suspensions containing P940 carbon nanotubes at $0.125,0.25$ and 0.5 wt\% were prepared utilising two different dispersion techniques, in order to evaluate the relationship between the state of dispersion and electrical conductivity. The first batch was ultrasonicated up to a total energy input of $1300 \mathrm{~J} / \mathrm{g}$, whilst the other was shear mixed at 1800 rpm up to 72000 cycles. Practical details of the ultrasonication procedure are given elsewhere [7]. These loadings were chosen since they can result in suspensions with appropriate viscosity for subsequent RTM infusion. The suspensions were placed in a freezer at $-18^{\circ} \mathrm{C}$ immediately following the dispersion process.

\subsection{Resin transfer moulding}

Moulding was carried out using an Isojet piston driven RTM setup. Twelve layers of fabric were laid up on the mould cavity in the $0^{\circ}$ direction in order to attain a final composite theoretical fibre volume fraction of approximately $57 \%$ and $59 \%$, for carbon and glass fibre composites respectively.

Each resin suspension was heated up to $40^{\circ} \mathrm{C}$ in order to reduce its viscosity and facilitate the diffusion of gases and moisture from the resin during the subsequent degassing in a vacuum chamber for 20 - $30 \mathrm{~min}$. After degassing the hardener was added to the resin and the mixture stirred for two minutes. A final degassing step was then performed, for a period of no longer than $10 \mathrm{~min}$, followed immediately by pouring the resin mixture into the preheated $\left(30^{\circ} \mathrm{C}\right)$ piston chamber of the RTM setup. Mould filling was performed under 2 bar pressure, into an aluminium tool with a glass top cover, allowing visual monitoring of the flow front position during injection. The mould temperature at injection was set at $65^{\circ} \mathrm{C}$ for all systems except the control and the P940 filled composite panels for which a mould temperature of $75^{\circ} \mathrm{C}$ was utilised. The cure cycle for all systems was $90 \mathrm{~min}$ at $80{ }^{\circ} \mathrm{C}(1 \mathrm{bar})$. The $340 \times 340 \mathrm{~mm}$ panels produced were then postcured in an oven at $140^{\circ} \mathrm{C}$ for $8 \mathrm{~h}$. 


\subsection{Electrical conductivity}

The set up utilised for AC impedance spectroscopy of liquid samples at room temperature consists of two solid $(\varnothing=25 \mathrm{~mm})$ copper cylindrical electrodes with adjustable gap. The sample is placed on one of the cylinders and the second cylinder comes in contact with the sample by moving to the prescribed gap position. Excess fluid is removed from the sides of the assembly once the second cylinder is fixed in position. The AC conductivity $(\sigma)$ dependence with temperature $(T)$ of the liquid unmodified resin was measured by immersing an interdigitated copper sensor (GIA, Pearson Panke Ltd) in a glass tube containing the liquid resin and placing it in an oven. In this case the conductivity was calculated based on the low frequency part of the real impedance spectrum, where the constant of calibration was taken from [24]. For both systems, the two electrodes are connected with a Solartron SI 1260 frequency response analyser that allows measurement of the complex impedance of the sample as a function of frequency. Twenty five frequencies, from $1 \mathrm{~Hz}$ to $1 \mathrm{MHz}$, were swept on a logarithmic scale. The electrical resistance of the samples was determined from the peak value of the imaginary impedance spectrum as described in a previous publication [25] and the electrical conductivity was computed using the resistance and the geometrical characteristics of the cell.

The conductivities of cured samples were measured on rectangular specimens, $30 \times 30 \times$ $3.2 \mathrm{~mm}$ for carbon composites and $30 \times 30 \times 3.7 \mathrm{~mm}$ for glass composites. The surfaces of interest were painted with a suspension of silver in methyl isobutyl ketone (Acheson Electrodag $1415 \mathrm{M}$ ) which allow good electrical contact between the surfaces of the sample and copper electrodes. The DC electrical resistivity of carbon composite samples was measured using a DC precision current source (Keithley model 6220) and a nanovoltmeter (Keithley model 2182A) via the three-point delta current reversal technique. The measurements were performed in three directions (through thickness, transverse and longitudinal) using a current of $100 \mu \mathrm{A}$. Through thickness impedance spectroscopy measurements of the glass composite samples were carried out using the frequency response analyser in a configuration similar to that used for the liquid suspension measurements. 


\subsection{Microscopy}

The state of dispersion of the CNTs in the epoxy resin at any given stage was assessed by transmission optical microscopy using an Olympus $\mathrm{BH}-2$ microscope. A small droplet of the liquid suspension was placed between a microscope glass slide and a cover slip, giving a sample thickness of $20 \pm 10 \mu \mathrm{m}$.

Resins containing P940 and C100 MWCNTs were cured to thickness of about $50 \mu \mathrm{m}$, between glass slides, with a cure regime identical to that applied to the bulk composite samples, for examination by transmission optical microscopy. Cryo-fracture surfaces of bulk cured nanocomposites and delamination fracture surfaces of the fibre composite were investigated using scanning electron microscopy (SEM).

\subsection{Rheology}

Samples of the suspensions utilised for RTM were collected for rheological measurements. A Bohlin CVO rheometer with 40/40 mm cone and plate geometry was used in steady shear mode, at the corresponding mould temperature. The viscosity of the suspensions was measured in the 0.1 to $1000 \mathrm{~s}^{-1}$ shear rate range.

\section{Results and discussion}

\subsection{Dispersion and electrical properties of MWCNT suspensions}

The effects of different mixing processes on the dispersion state and the electrical conductivity of the liquid samples were investigated, as basis for the selection of adequate suspensions to be utilised in the infusion of carbon and glass reinforcements. Fig. 2 illustrates the evolution of microstructure associated with the dispersion of 0.25 wt $\%$ P940 MWCNTs in the 
epoxy resin by means of ultrasonication. Similar evolution of the dispersion microstructure was observed for the suspensions filled with $0.125 \mathrm{wt} \%$ and $0.5 \mathrm{wt} \%$ P940 CNTs. Fig. 3 summarises the evolution of electrical conductivity of the suspensions during dispersion processing. Highly entangled large and isolated MWCNTs aggregates with well defined boundaries characterise the initial resin suspension (Fig. 2(a)). As the total ultrasonic energy input to the suspension increases, the aggregate boundaries become blurred as CNTs gradually unravel forming areas of separate and individual CNTs around the aggregate dense core (Fig. 2(b)). Although dimensionally large aggregates are still present at intermediate levels of dispersion such as those shown in Fig. 2(b), a significant increase of the electrical conductivity of the suspension is observed (Fig. 3), which implies that at this stage, the number of conductive links formed increases considerably. Further ultrasonication of the suspension, up to $1300 \mathrm{~J} / \mathrm{g}$, results in a suspension characterised predominantly by a homogenous distribution of carbon nanotubes where few dense aggregates with a maximum dimension of up to $50 \mu \mathrm{m}$ can be observed.

The electrical conductivity of all ultrasonicated suspensions rises sharply after the initial stages of ultrasonication. After $350 \mathrm{~J} / \mathrm{g}$ and $200 \mathrm{~J} / \mathrm{g}$ of processing, the conductivity of suspensions filled with 0.5 wt $\%$ and 0.25 wt $\%$ P940, and 0.125 wt $\%$ P940, respectively, becomes approximately constant until the end of the process (Fig. 3). Higher electrical conductivity results are associated with efficient dispersions containing increasing content of CNTs. However, the electrical conductivity results for $0.25 \mathrm{wt} \%$ and $0.5 \mathrm{wt} \% \mathrm{P} 940$ suspensions dispersed by ultrasonication are similar. Considerably smaller electrical conductivity, of about one order of magnitude, is obtained for the suspension containing $0.125 \mathrm{wt} \%$ P940 CNTs when processed by ultrasonication. High shear mixing leads to distinct conductivity results for 0.5 and $0.25 \mathrm{wt} \%$ CNT loaded suspension during intermediate stages of dispersion, but at the final stage of dispersion (72000 cycles) both suspensions reach identical conductivity values.

The overall effect of ultrasonication process is a step change of electrical conductivity of the suspension by nearly three orders of magnitude. In contrast, the electrical conductivity of the high shear mixed liquid suspensions shows an increase by two orders of magnitude (Fig. 3). These results indicate that ultrasonication is a more effective route of dispersing unmodified 
nanotubes in the epoxy system of this study. An electrical conductivity plateau region is observed for ultrasonication energy inputs between $550 \mathrm{~J} / \mathrm{g}$ and $1.3 \mathrm{~kJ} / \mathrm{g}$ for both 0.25 and 0.5 wt\% loaded suspensions. Consequently, for RTM manufacturing purposes a total energy input of $800 \mathrm{~J} / \mathrm{g}$ was chosen to disperse P940 CNTs in the epoxy resin at $0.25 \mathrm{wt} \%$ CNT loading, since $0.5 \mathrm{wt} \%$ CNT contents lead to extremely viscous suspensions for liquid moulding. Suspensions with lower CNT contents, such as $0.125 \mathrm{wt} \%$, were not considered for infusion due to the considerably lower electrical conductivity measured for the liquid suspension, when compared to $0.25 \mathrm{wt} \%$ and $0.5 \mathrm{wt} \% \mathrm{P} 940 \mathrm{CNT}$ suspensions.

Fig. 4 shows SEM micrographs of the fracture surfaces of P940 cured nanocomposites, used to evaluate the combined effect of ultrasonication and cure on the final morphology of the carbon nanotube network. Similarly to the optical microscopy results, large and dense agglomerates are present in the unprocessed resin (Fig. 4(a)). At the end of ultrasonication (1.3 $\mathrm{kJ} / \mathrm{g}$ ) a considerable reduction of the average size of CNT aggregates and of the number of aggregates larger than $50 \mu \mathrm{m}$ is observed (Fig. 4(b)). Transitional areas between dense aggregates and dispersed CNTs are characteristic of the nanocomposite and can be seen in Fig. 4(d). These areas represent the unravelling of the CNTs at the boundaries of the dense aggregates with the resin. These unravelled CNTs eventually become loose and spread into the resin. Fig. 4(e) and (f) are high magnification images of CNT aggregates and represent the different morphology of dense and loose aggregates within the resin before and after the ultrasonication, respectively. Dense aggregates are characterised by a higher level of CNT entanglement and areal density, which is evidenced by the close proximity between individual CNTs. In contrast, in loose aggregates, as the one shown in Fig. 4(f), the mean distance between CNTs appears larger.

Fig. 5 summarises the liquid electrical conductivity values for the various suspension formulations of this study. Dispersion processing of the C100 nanotube system by triple roll milling and high shear mixing leads to an increase in conductivity by two orders of magnitude, as shown in Fig. 5. Further processing by ultrasonication causes a slight reduction in the electrical conductivity of the suspension, which may be explained by damage (reduction of the 
aspect ratio) of the CNTs during ultrasonication; indeed Fig. 7 indicates a finer structure of loose aggregates in the ultrasonicated material. The sharp reduction in conductivity observed when the suspension of $\mathrm{C} 100$ nanotubes was heated up for $30 \mathrm{~min}$ at $40^{\circ} \mathrm{C}$ (Fig. 5) is thought to be due to reaggregation of the carbon nanotubes, accelerated by the elevated temperature. Such reaggregation could disrupt the conductive network formed by the nanotubes during dispersion processing. However, the conductivity remains significantly higher than of the original suspension which indicates that the reaggregated agglomerates of nanotubes exhibit a looser structure compared to the aggregates of the material prior to dispersion. Potential changes in the unmodified resin conductivity during processing were investigated. The conductivity of the liquid prepolymer at room temperature remains unchanged after a thermal cycle similar to that applied to the C100 suspension (Fig. 6 (a)). In contrast, significant changes in conductivity occur during the cure and post cure of the two-component system (Fig. 6 (b)). The conductivity increases significantly during heating up before the first dwell and between cure and post cure as a result of the increased mobility of charge carriers at higher temperature. During the isothermal segment of the cure/post cure profile the conductivity decreases as a consequence of cross linking and the corresponding mobility limitations the forming network creates for charge carrying entities [26].

The electrical conductivity of the initial C100 suspension is lower than that of the corresponding P940 suspension, which is consistent with the lower average aspect ratio for the C100 system. Micrographs of the surface modified MWCNT filled suspension are shown in Fig. 8, characterised by a stable and uniform dispersion of CNTs throughout the resin. Relatively small and loose aggregates and individual carbon nanotubes coexist in suspension, surrounded by areas of clear epoxy resin. The suspension conductivity of the surface modified system is low and comparable with the conductivity of the C100 system. This can be attributed to the limited tendency of the surface modified system to aggregate and form a conductive network and the effect of surface modification to the conductivity of the network of CNTs.

The phenomenon of cure-induced reaggregation was investigated for the cases of C100 and P940 nanotubes, as shown in Fig. 9. Reaggregation observed in both materials was manifested 
as an increase in the amount and size of clear resin areas and by the formation of relatively denser (darker) aggregates.

\subsection{RTM adaptation and processing issues in manufacture of multiscale composites}

For simplicity, pseudo-unidirectional uniweave fabrics were selected as reinforcement. This avoids complications in processing that could result from multiple fibre orientation as the permeability of the fabrics and the rate of filtration of nanoparticles is expected to be uniform across the thickness. Also, the electrical properties measured after infusion and curing correspond to a single fibre orientation, allowing an evaluation of the effects of carbon nanotube addition to be made.

Compared to normal composite processing, RTM infusion with carbon nanoparticle filled resins requires process modification in respect of the degassing steps as well as selection of the appropriate mould filling temperatures to compensate for the increased viscosity of the resin. CNT reaggregation during degassing was observed in the case of the P940/epoxy system. High suspension viscosity at the standard degassing temperature of $40^{\circ} \mathrm{C}$ for this system resulted in unacceptably long degassing process. Ultimately the suspension was heated up to $50^{\circ} \mathrm{C}$ to reduce the suspension viscosity sufficiently to allow efficient removal of volatiles. The duration of this process was approximately one hour and the dispersion state was compromised as a consequence, with slightly larger aggregates present in the sample after degassing. In the case of the $\mathrm{C} 100$ system as well as of the surface modified material changes to the procedure of resin degassing were not required, as the lower viscosity of these systems allowed the process to be completed at $40{ }^{\circ} \mathrm{C}$ within a relatively short time.

These results and the results on the electrical response of nanocomposites presented in the previous section point to the difficulties arising due to the interplay between the state of dispersion, processability and electrical performance. Dispersion states corresponding to high conductivities such as these observed in the P940 nanocomposite are inherently more difficult to degas and require modification of the degassing cycle usually involving higher temperatures 
and/or longer process times. A balance between the benefits of degassing and the loss of electrical performance due to extensive reaggregation is necessary when designing the modified process.

\subsubsection{Rheology}

Fig. 10 shows the viscosity dependence on the shear rate for all the suspensions utilised for liquid moulding at the corresponding mould temperature. Shear rate dependent suspensions such as the ones filled with unmodified CNTs show higher viscosities. In contrast, the unfilled and the surface modified CNT filled epoxy behave linearly and have lower viscosity values. The viscosities of the P940 system are the highest observed at low and intermediate strain rates. This result is consistent with the observations during degassing where modification of the schedule is required to complete the process for this system. As the shear rate increases the viscosity of all systems tends to plateau to a level that is similar to that of the unfilled epoxy.

\subsubsection{Filtration}

The solution of Darcy's problem for a fluid of constant viscosity $\eta$, in a porous medium of constant effective permeability $K$ and porosity $\varepsilon$ in an one dimensional situation with prescribed pressures at the inlet and outlet can be described by the following expression:

$$
h(t)=\sqrt{\frac{2 K}{\varepsilon \eta}\left(p_{o}-p_{\infty}\right) t}
$$

Here $h(t)$ denotes the flow front position at time $t$, and $p_{o}$ and $p_{\infty}$ are the prescribed pressures at the inlet and outlet of the mould respectively. This solution implies a linear dependence of flow front position on the square root of time.

Fig. 11 illustrates the resin flow front position versus the square root of time alongside a linear fit using Eq. (1). Significant deviation from linear behaviour is observed in both cases of 
unmodified CNTs. In contrast, the control and surface modified carbon nanotubes filled material follow the linear fit closely. Deviation from the square root dependence can be attributed both to the highly non Newtonian behaviour of the modified resin and the retention of nanoparticles by the reinforcement which modifies both the local permeability of the fabric and the resin viscosity. Longer filling times were also characteristic of the two unmodified CNT filled materials. This can be attributed to their intrinsically higher viscosity profiles and the reduction of the reinforcement porosity caused by the clogging of the available pores through which resin circulates.

Fig. 12 shows the resin flow front during RTM injection together with a close view of the flow front where inter-tow and intra-tow flow can be distinguished, for the case of C100 CNTs and glass fabric. CNT filtration was observed macroscopically for the systems containing unmodified CNT. Resin was seen running clear at the outlet gate for the injection of carbon reinforcement composite with P940 filled suspension. In glass reinforced composites infused with C100 filled epoxy some colour variation in the final composite was a clear indication of CNT filtration by the porous media. In contrast, surface modified CNTs did not show macroscopic and qualitative evidence of filtration, since resin was not seen running clearer at the outlet gate.

The distribution of CNTs at the inlet and outlet regions, and on delamination surfaces of the composite panel were investigated for all the systems by SEM. Very large aggregates $(\sim 50 \mu \mathrm{m})$ were found at the inlet for the case of P940 CNTs. A partial view of one of these aggregates is shown in Fig. 13 (a). The composite structure incorporates two pore scales through where resin flow occurs $(100 \mu \mathrm{m}$ and $10 \mu \mathrm{m})$. These are characteristic of the inter and intra-tow spacing dimensions. Large and highly entangled CNT aggregates concentrated in resin rich pockets regions, located between the carbon tows and the orthogonal stitches that hold the tows together, are observed on the composite delamination surface of the P940 material in Fig. 13 (c) and (d). These aggregates become less frequent away from the resin inlet due to filtration. Furthermore, the resin located at the outlet region contains virtually no CNT agglomerates as shown in Fig. 13 (b). These observations indicate the occurrence of cake filtration at a microscopic level. Size exclusion is considered as the main filtration mechanism for this CNT system. In addition, the non-Newtonian behaviour of the suspension associated to 
hydrodynamic effects, caused by a non-uniform shear field during fluid flow and nonsphericity of the particles [27], can also contribute to preferential particles deposition especially in stagnation zones where low shear strains are present. Though not observed in SEM, other potential mechanisms of filtration in fibrous media for CNT filled suspensions such as inertial effects and direct interception with the fibre surface could be active.

C100 nanotubes were also filtered when passing through the glass porous media although smaller and looser aggregates (10 $\mu \mathrm{m}$ maximum dimension) than the ones seen in P940 filled resin were present at the inlet region as illustrated in Fig. 14. Also small and loose CNT aggregates were found in resin rich pockets and inter-fibre areas of the composite as shown on

Fig. 14 (c). In contrast to the P940 system where cake filtration is observed, the gradient in nanotube concentration in the C100 system is significantly lower which indicates that deep bed filtration is the dominant mechanism in this system in the initial stage of filling. Later in the process the accumulation of retained nanoparticles might lead to retention via size exclusion and 'microscopic' cake filtration.

The potential reaggregation of CNTs is a significant factor in the interaction between flow and filtration in unmodified systems such as the P940 and C100 nanotubes. The reaggregation would lead to gradually increased size of filtered nanoparticles and eventually promote cake filtration. This effect is not present in modified systems like the surface modified nanotubes used in this study. This fact in combination with the small initial aggregate size leads to negligible filtration as evidenced by the uniform presence of 2-3 $\mu \mathrm{m}$ size loose aggregates both at the inlet and outlet regions of surface modified CNT panels as illustrated in Fig. 15.

\subsection{Electrical properties of the CNT modified fibre composites}

The results of electrical measurements of carbon laminates indicate highly anisotropic electrical behaviour of the composite as shown in Fig. 16 (a) and (b). The electrical conductivity in the fibre direction is two to three orders of magnitude higher that the conductivity in the inplane transverse and through thickness directions respectively. This is expected as the carbon 
fibres form continuous conductive paths in the unidirectional material, governing the conductivity in this direction. The drop in the longitudinal electrical conductivity when adding CNTs to the composite is due to the scatter of results. The conductivity in the in-plane transverse direction is one order of magnitude higher than that in the through thickness direction. In this case the level of contact between adjacent carbon fibres is the governing parameter and the packing of fibres in the in-plane direction is denser than that between successive layers in the out-of-plane direction. Consequently, the addition of carbon nanotubes has an effect only in the conductivity of the through thickness direction of carbon composites where the electrical behaviour of resin rich areas plays a more significant role. The improvement in through thickness conductivity of carbon laminates is more significant for the unmodified CNTs (P940) than the surface modified system due to the tendency of unmodified nanotubes to form a conductivity network via reaggregation. However, the scattering in conductivity values is higher in the unmodified system which is indicative of non-uniformity caused by filtration in this material.

The addition of $\mathrm{C} 100$ carbon nanotubes in the glass composite also increases the through thickness conductivity of the glass composite. The increase is relatively modest (approximately one order of magnitude), a result which is in line with the relatively low conductivity of the $\mathrm{C} 100$ suspension reported in Fig. 5. Similarly to the unmodified CNT system used with carbon fibres, the conductivity of the CNT loaded material exhibits high scatter in its values, as a result of filtration effects.

Filtration is also investigated in Fig. 16 (c), by identifying spatial variations of through thickness conductivity of carbon reinforced composites. A slight reduction of the electrical conductivity of the composite can be observed. This phenomenon occurs even in the case of surface modified CNTs indicating filtration at the microscopic level.

\section{Concluding remarks}

The outcome of this investigation demonstrates the feasibility of processing realistic aerospace grade composites, comprising aerospace materials, such as epoxy resin and carbon 
fibre fabric at high fibre volume fraction, with CNT loaded epoxy matrices, resulting in enhanced electrical performance. A range of possibilities exists for the design of the process and the selection of materials.

The modification of epoxy resins by carbon nanotubes has for consequence the need to modify the process routes for the manufacture of multiscale composite laminates via liquid resin moulding processes. A dispersion step needs to be added before the filling process during which the nanotubes are suspended in the liquid resin with the aim of maximising their eventual performance enhancement effects. The state of dispersion can be evaluated successfully by carrying out measurements of electrical conductivity during the dispersion process. In the formulations tested here it was found that additional dispersion processing was only necessary for unmodified carbon nanotubes which enter the process in an aggregated form and also have the tendency to reaggregate during processing. Electrical conductivity measurements have shown that ultrasonication is a better way of dispersing P940 CNTs when compared to high shear mixing. P940 contents of 0.5 wt $\%$ were found to cause just a slight improvement in the electrical conductivity of the suspension when compared to $0.25 \mathrm{wt} \%$, whilst lower contents, such as $0.125 \mathrm{wt} \%$ led to suspensions with lower electrical conductivity by about one order of magnitude, not offering as much potential in enhancing the final composite conductivity as the ones containing $0.25 \mathrm{wt} \%$ and $0.5 \mathrm{wt} \%$ CNTs. In addition, the higher viscosity of the $0.5 \mathrm{wt} \%$ suspension was found to be inappropriate for RTM. Therefore, the CNT loading for all the systems was kept at $0.25 \mathrm{wt} \%$.

Degassing schedules for well dispersed unmodified CNT-containing systems need to be carried out at elevated temperatures, to compensate for the increase in viscosity. This process modification is not straightforward as the increase in temperature also accelerates reaggregation effects that could potentially degrade the efficiency of nanotube addition with respect to electrical performance and can also initiate the curing reaction and increase the viscosity of the system further. The increase in viscosity also plays a role during the filling stage of liquid moulding processes by slowing down the progression of the flow front. 
Surface modified nanotubes offer an easy processing solution, requiring minimal process modifications but at the cost of higher material cost and lower achievable electrical conductivity compared to the composites made with unmodified multiwalled nanotubes. Unmodified nanotubes require adaptations both in the degassing and filling stages of the RTM process. The cost of these needs to be set against the higher raw material cost of unmodified tubes and higher potential for enhancement of the electrical behaviour.

The interaction of suspended and aggregated nanotubes with the reinforcement fibres results in filtration effects that can be significant in unmodified CNT- containing systems. The state of dispersion of the nanoparticles and their tendency to reaggregate governs the type of filtration behaviour. 'Microscopic' cake filtration dominates in situations where aggregates with sizes comparable to the pores of the reinforcement are present in the suspension. In these systems relatively large CNT aggregates are found in resin rich-pockets closer to the inlet side of the mould, whereas very few nanoparticles are present in the outlet side of the composite. In contrast deep bed filtration occurs in situations where the size of aggregates remains relatively small compared with the pores. Filtration effects affect the macroscopic resin flow in the mould due to the dependence of viscosity and permeability on the local concentration of nanoparticles as well as the modification of porosity with the accumulation of retained particles on the fabric.

Both carbon and glass fibre reinforced composites containing the CNT-filled matrix were prepared, resulting in fibre volume fractions of $57 \%$ and $59 \%$ respectively. The enhancement of electrical behaviour by the addition of CNTs in carbon composites followed the anisotropic character of these materials. The through-thickness conductivity almost doubled with the addition of nanotubes and the enhancement was stronger for unmodified nanotubes. The conductivity in the in-plane directions, which is dominated by that of the carbon fibre, remained unaffected. The electrical conductivity of glass composites increased by one order of magnitude with the addition of nanotubes, to a level which can still be considered modest given the very low conductivity of the unfilled glass composite. Electrical conductivity measurements of specimens at different locations in the mould were found useful to evaluate the extent of CNT filtration during injection, especially for the case of surface modified CNTs which was not 
identified both macroscopically and microscopically. Despite the larger extent of filtration verified for the unmodified CNT system, these CNTs provide a better improvement of the composite electrical conductivity properties when compared to surface modified CNTs.

The phenomenon of filtration plays an important role in the morphology of the final multiscale composite, with high filtration effects observed in injection of material with unmodified nanotubes, leading to significant gradients in nanofiller concentration. This naturally leads to gradients of properties, especially electrical conductivity, in the cured component. Although this can be considered a problem, it could also be seen as an opportunity offered by the possibility of introducing high CNT loading into critical areas of the component where high electrical conductivity is required. 


\section{Acknowledgements}

The work reported here was funded by the Engineering and Physical Sciences Research

Council via the Innovative Manufacturing Research Centre at Cranfield University (GR/R68139/01) and by BAE Systems. The authors acknowledge J W Treiber for helpful assistance in commissioning the new RTM equipment. 


\section{References}

[1] Gojny FH, Wichmann MHG, Fiedler B, Kinloch IA, Bauhofer W, Windle AH, et al. Evaluation and identification of electrical and thermal conduction mechanisms in carbon nanotube/epoxy composites. Polymer. 2006;47(6):2036-2045.

[2] Bauhofer W, J.Z. K. A review and analysis of electrical percolation in carbon nanotube polymer composites. Compos Sci Technol. 2009;69(10):1486-1498.

[3] Fiedler B, Gojny FH, Wichmann MHG, Nolte MCM, Schulte K. Fundamental aspects of nanoreinforced composites. Compos Sci Technol. 2006;66(16):3115-3125.

[4] Thostenson ET, Ren Z, Chou T-W. Advances in the science and technology of carbon nanotubes and their composites: a review. Compos Sci Technol. 2001;61(13):1899-1912.

[5] Fiedler B, Gojny FH, Wichmann M, Bauhofer W, Schulte K. Can carbon nanotubes be used to sense damage in composites? Ann chim. 2004;29(6):81-94.

[6] Advani SG. Processing and properties of nanocomposites. Singapore: World Scientific Publishing Co Pte Ltd; 2007.

[7] Battisti A, Skordos AA, Partridge IK. Monitoring dispersion of carbon nanotubes in a thermosetting polyester resin. Compos Sci Technol. 2009;69(10):1516-1520.

[8] Cooper CA, Ravich D, Lips D, Mayer J, Wagner HD. Distribution and alignment of carbon nanotubes and nanofibrils in a polymer matrix. Compos Sci Technol. 2002;62(7-8):1105-1112.

[9] Bekyarova E, Thostenson E, Yu A, Kim H, Gao J, Tang J, et al. Multiscale carbon nanotubecarbon fiber reinforcement for advanced epoxy composites. Langmuir. 2007;23(7):3970-3974. [10] Fan Z, Hsiao K-T, Advani SG. Experimental investigation of dispersion during flow of multiwalled carbon nanotube/polymer suspension in fibrous porous media. Carbon. 2004;42(4):871876.

[11] Gojny FH, Wichmann MHG, Fiedler B, Bauhofer W, Schulte K. Influence of nanomodification on the mechanical and electrical properties of conventional fibre-reinforced composites. Composites Part A 2005;36(11):1525-1535. 
[12] Böger L, Wichmann M, Meyer L, Schulte K. Load and health monitoring in glass fibre reinforced composites with an electrically conductive nanocomposite epoxy matrix. Compos Sci Technol. 2008;68(7-8):1886-1894.

[13] Qiu JJ, Zhang C, Wang B, Liang R. Carbon nanotube integrated multifunctional multiscale composites. Nanotechnology. 2007;18(27):1-11.

[14] Thostenson ET, Gangloff JJ, Li CY, Byun JH. Electrical anisotropy in multiscale nanotube/fiber hybrid composites. Appl Phys Lett. 2009;95(7):073111.

[15] Zhou Y, Pervin F, Lewis L, Jeelani S. Fabrication and characterization of carbon/epoxy composites mixed with multi-walled carbon nanotubes. Mater Sci Eng, A 2008;475(1-2):157165.

[16] Inam F, Wong DY, Kuwata M, Peijs T. Multiscale hybrid micro-nanocomposites based on carbon nanotubes and carbon fibers. J Nanomater. 2010;2010:1-12.

[17] Kim M, Park YB, Okoli OI, Zhang C. Processing, characterization, and modeling of carbon nanotube-reinforced multiscale composites. Compos Sci Technol. 2009;69(3-4):335-342.

[18] Gao L, Chou T-W, Thostenson ET, Godara A, Zhang Z, Mezzo L. Highly conductive polymer composites based on controlled agglomeration of carbon nanotubes. Carbon. 2010;48(9):2649-2651.

[19] Garcia EJ, Wardle BL, John Hart A. Joining prepreg composite interfaces with aligned carbon nanotubes. Composites Part A 2008;39(6):1065-1070.

[20] Wichmann MHG, Sumfleth J, Gojny FH, Quaresimin M, Fiedler B, Schulte K. Glass-fibrereinforced composites with enhanced mechanical and electrical properties - Benefits and limitations of a nanoparticle modified matrix. Eng Fract Mech. 2006;73(16):2346-2359.

[21] Fan Z, Advani SG. Capillary effect of multi-walled carbon nanotubes suspension in composite processing. J Nanosci Nanotechnol. 2008;8(4):1669-1678.

[22] Lefevre D, Comas-Cardona S, Binetruy C, Krawczak P. Coupling filtration and flow during liquid composite molding: Experimental investigation and simulation. Compos Sci Technol. 2009;69(13):2127-2134. 
[23] Lefevre D, Comas-Cardona S, Binétruy C, Krawczak P. Modelling the flow of particle-filled resin through a fibrous preform in liquid composite molding technologies. Composites Part $A$ 2007;38(10):2154-2163.

[24] Karkanas PI. Cure modelling and monitoring of epoxy/amine resin systems. Cranfield University, PhD thesis, 1997.

[25] Mijovic J, Yee CFW. Use of complex impedance to monitor the progress of reactions in epoxy/amine model systems. Macromolecules. 1994;27(25):7287-7293.

[26] Skordos AA, Partridge IK. Determination of the degree of cure under dynamic and isothermal curing conditions with electrical impedance spectroscopy. J Polym Sci, Part B. 2004;42(1):146-154.

[27] Herzig JP, Leclerc DM, Goff PL. Flow of suspensions through porous media - Application to deep filtration. Ind Eng Chem. 1970;62(5):8-35.

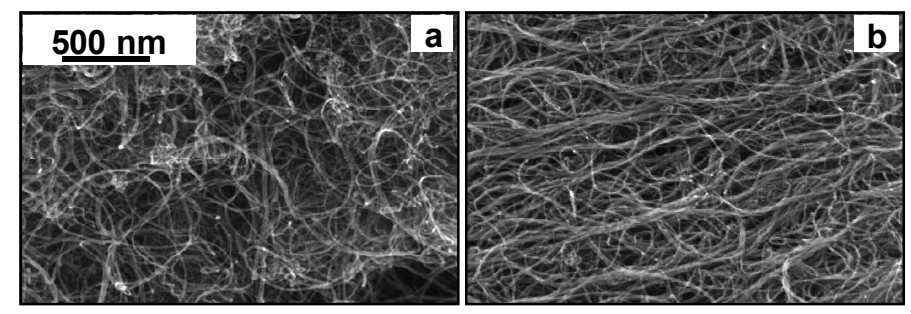

Fig. 1 SEM of as received P940 (a) and C100 unmodified MWCNTs (b).

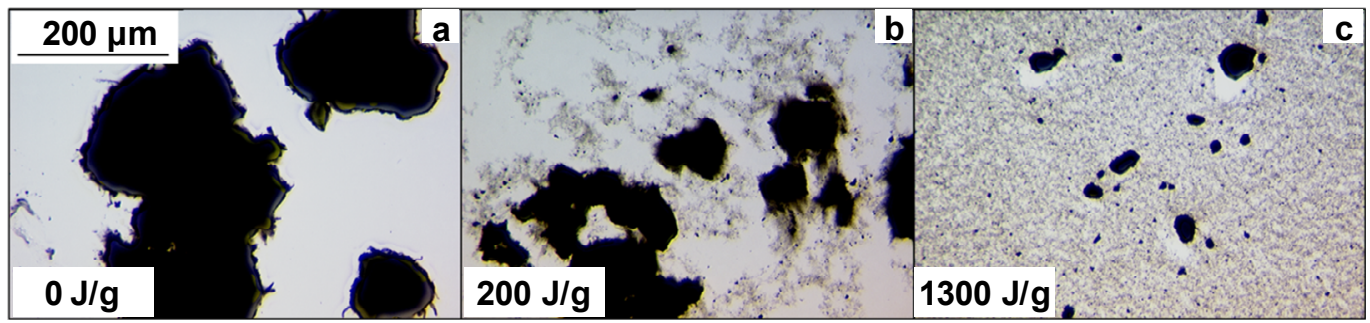


Fig. 2 Liquid state optical transmission micrographs of the $0.25 \mathrm{wt} \%$ P940 filled suspensions at different dispersion/processing levels: before ultrasonication (a); initial stages (b); after completion of the process (c).

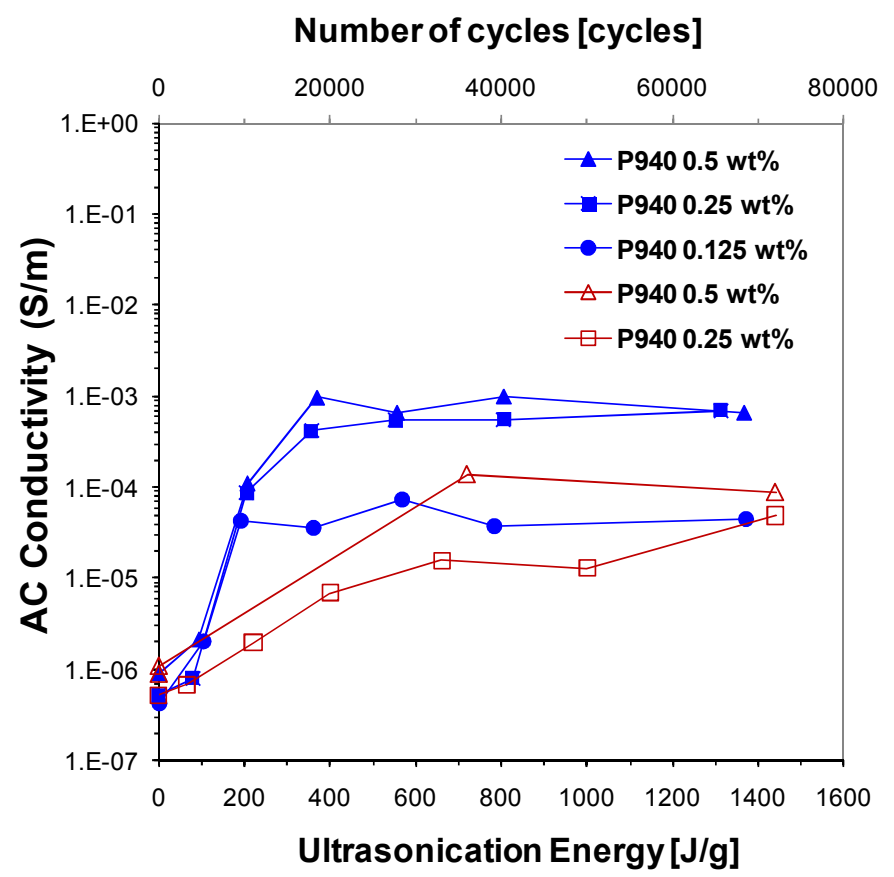

Fig. 3 Evolution of the AC electrical conductivity during ultrasonication and shear mixing of P940 filled suspensions (filled symbols represent ultrasonicated material, whilst open symbols represent high shear mixed suspensions). 


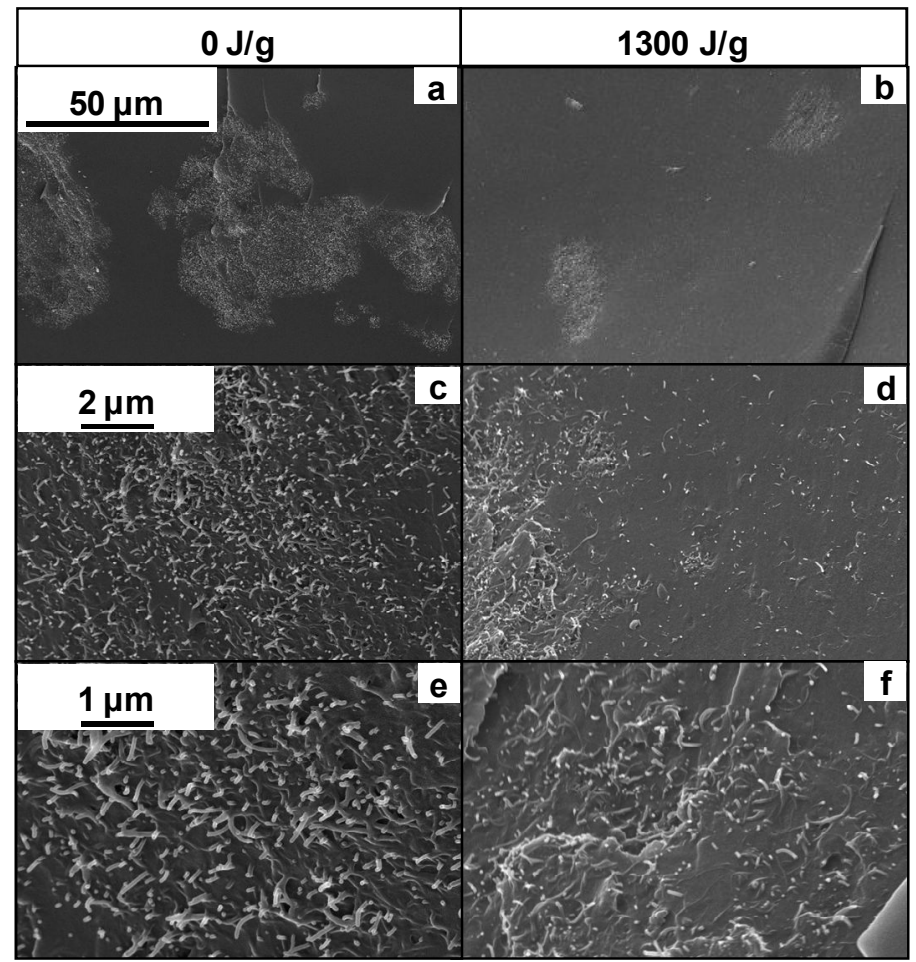

Fig. 4 SEM micrographs of fracture surfaces of P940/epoxy nanocomposites at the beginning ((a), (c) and (e)) and end ((b), (d) and (f)) of the ultrasonication process.

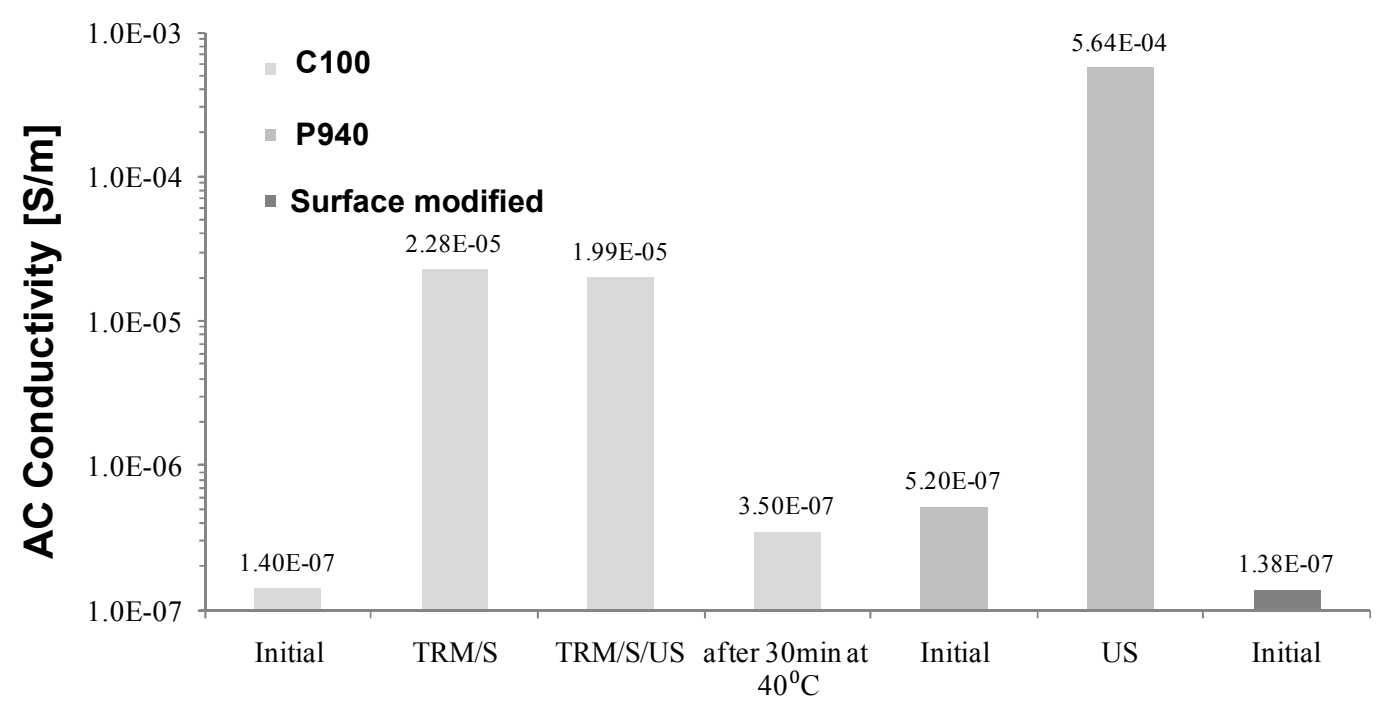

Fig. 5 Electrical conductivity of 0.25 wt $\%$ CNT filled liquid resins. 

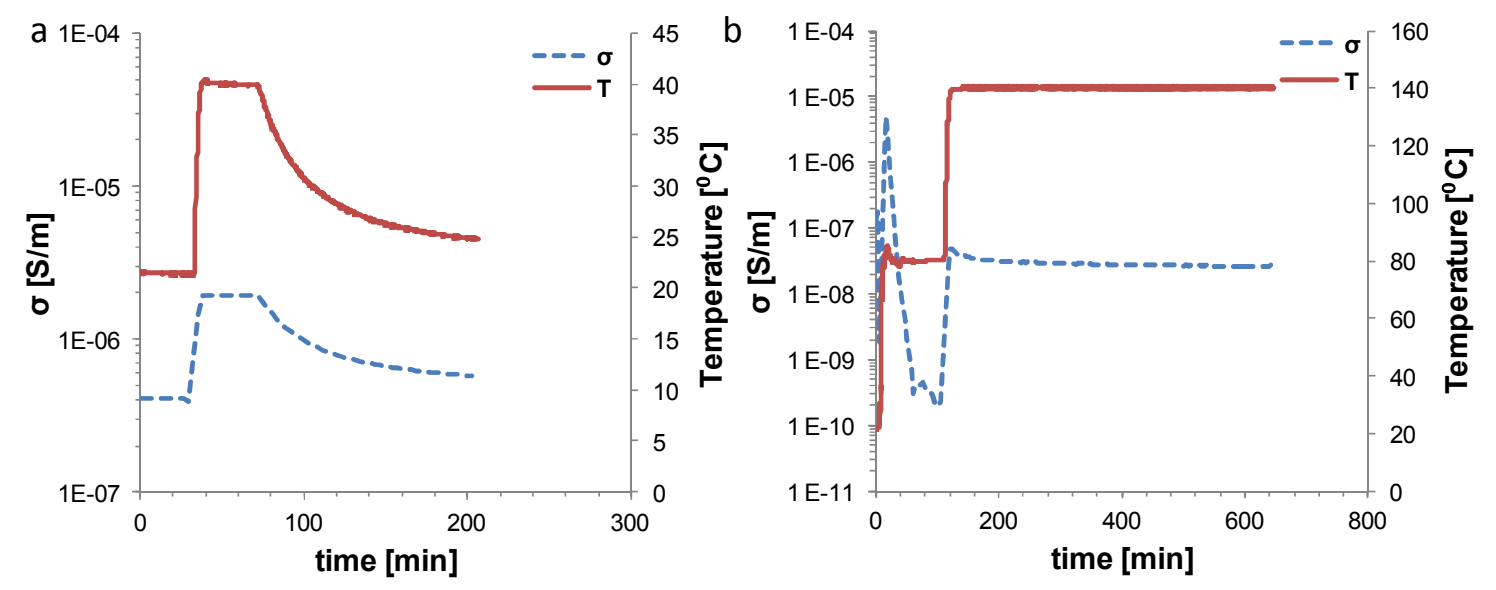

Fig. 6 Electrical conductivity of: the unmodified prepolymer during a thermal cycle similar to the one applied to $\mathrm{C} 100$ suspension (a); the two-component resin during cure and post-cure (b).

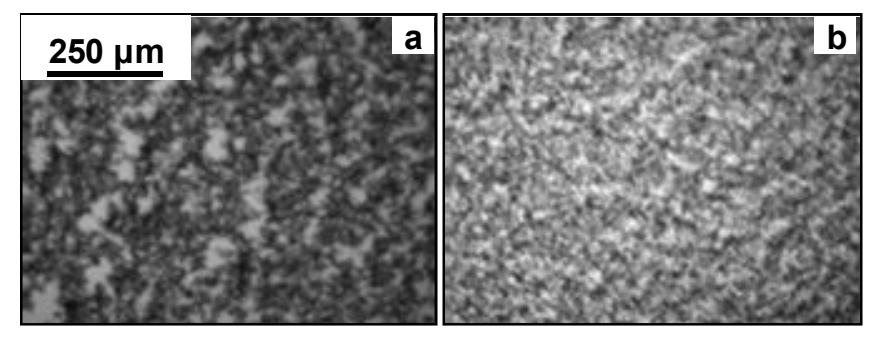

Fig. 7. Liquid state transmission optical micrographs of the $0.25 \mathrm{wt} \% \mathrm{C} 100$ filled suspensions at different dispersion steps: after triple roll milling and shear mixing (a) and subsequent ultrasonication $(650 \mathrm{~J} / \mathrm{g})(\mathrm{b})$.

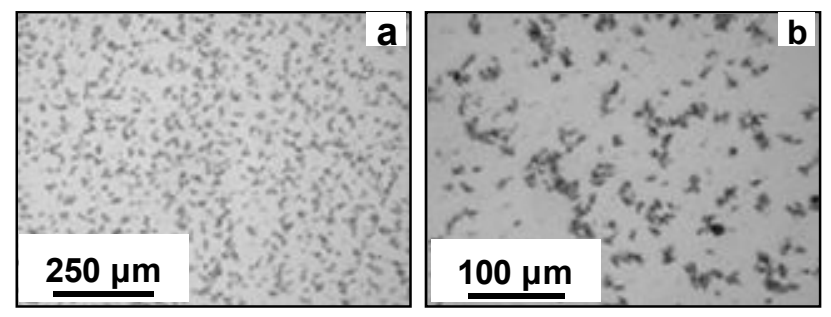

Fig. 8 Liquid state optical transmission micrographs of the $0.25 \mathrm{wt} \%$ surface modified CNT filled epoxy at low (a) and high (b) magnification. 


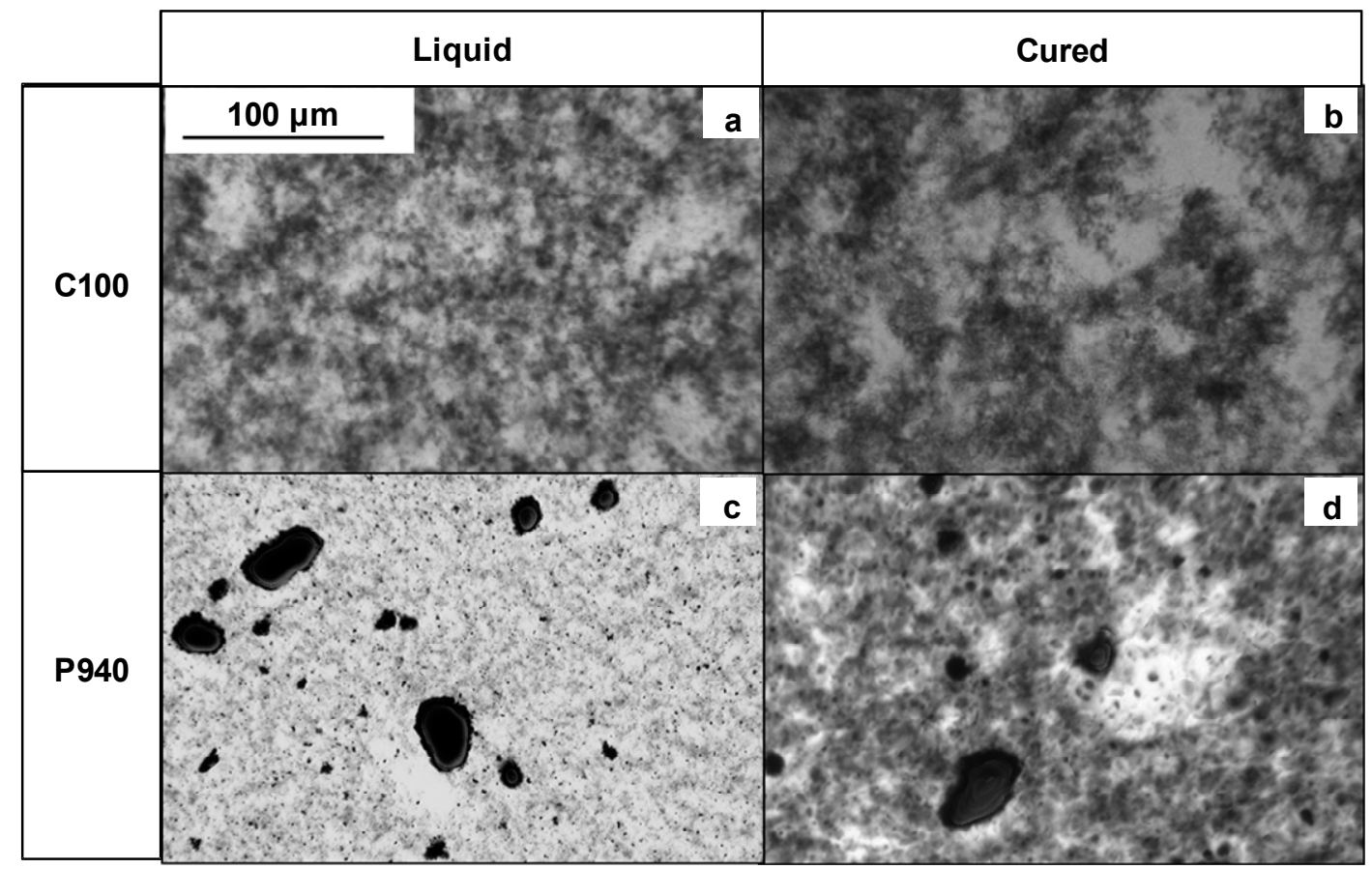

Fig. 9 Liquid and cured state transmission micrographs of 0.25 wt $\%$ C100 and P940 filled epoxy: liquid C100 system (a); cured C100 system (b); liquid P940 system (c); cured P940 system (d).

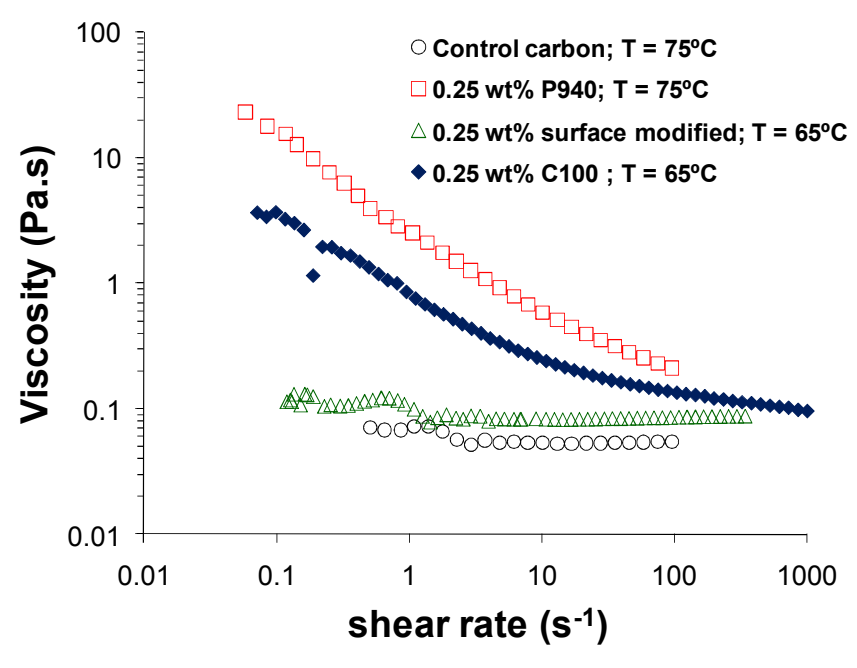


Fig. 10 Suspension viscosity as a function of shear rate (note that 'control carbon' refers to the viscosity of the reference resin utilised to infuse carbon fibre reinforcements).

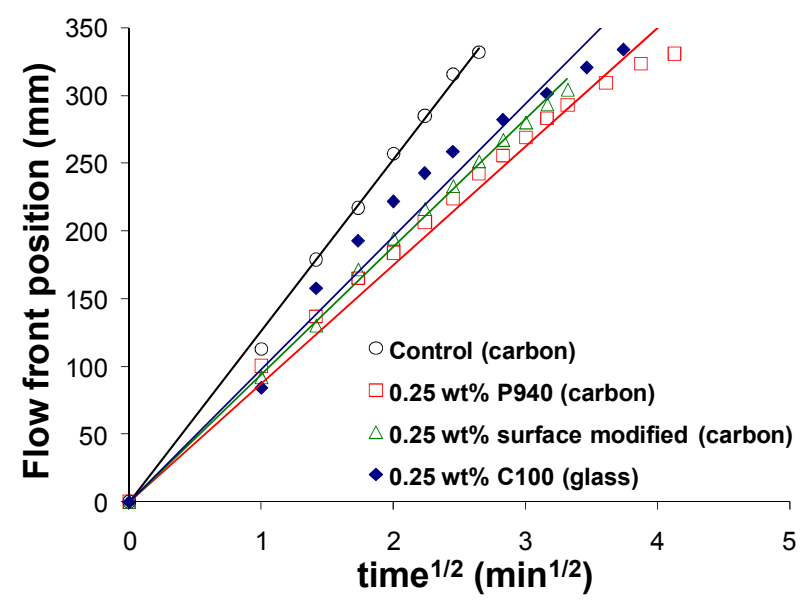

Fig. 11 Resin flow front profiles during RTM injection of carbon and glass reinforcements, as specified.

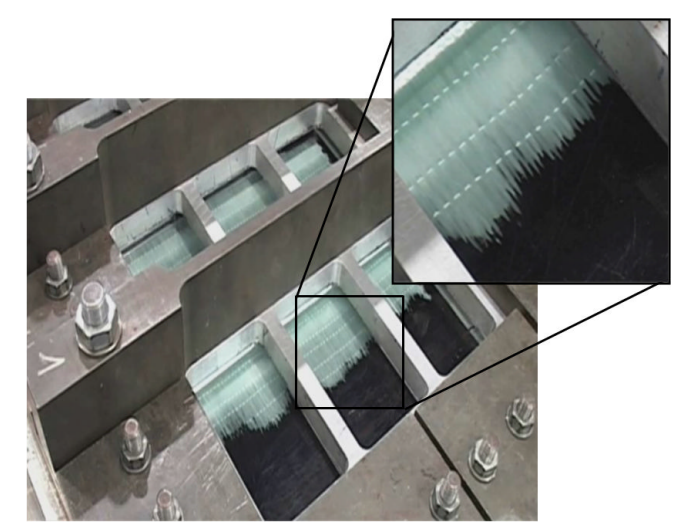

Fig. 12 Resin flow front during RTM injection of glass composite with C100 filled suspension. 


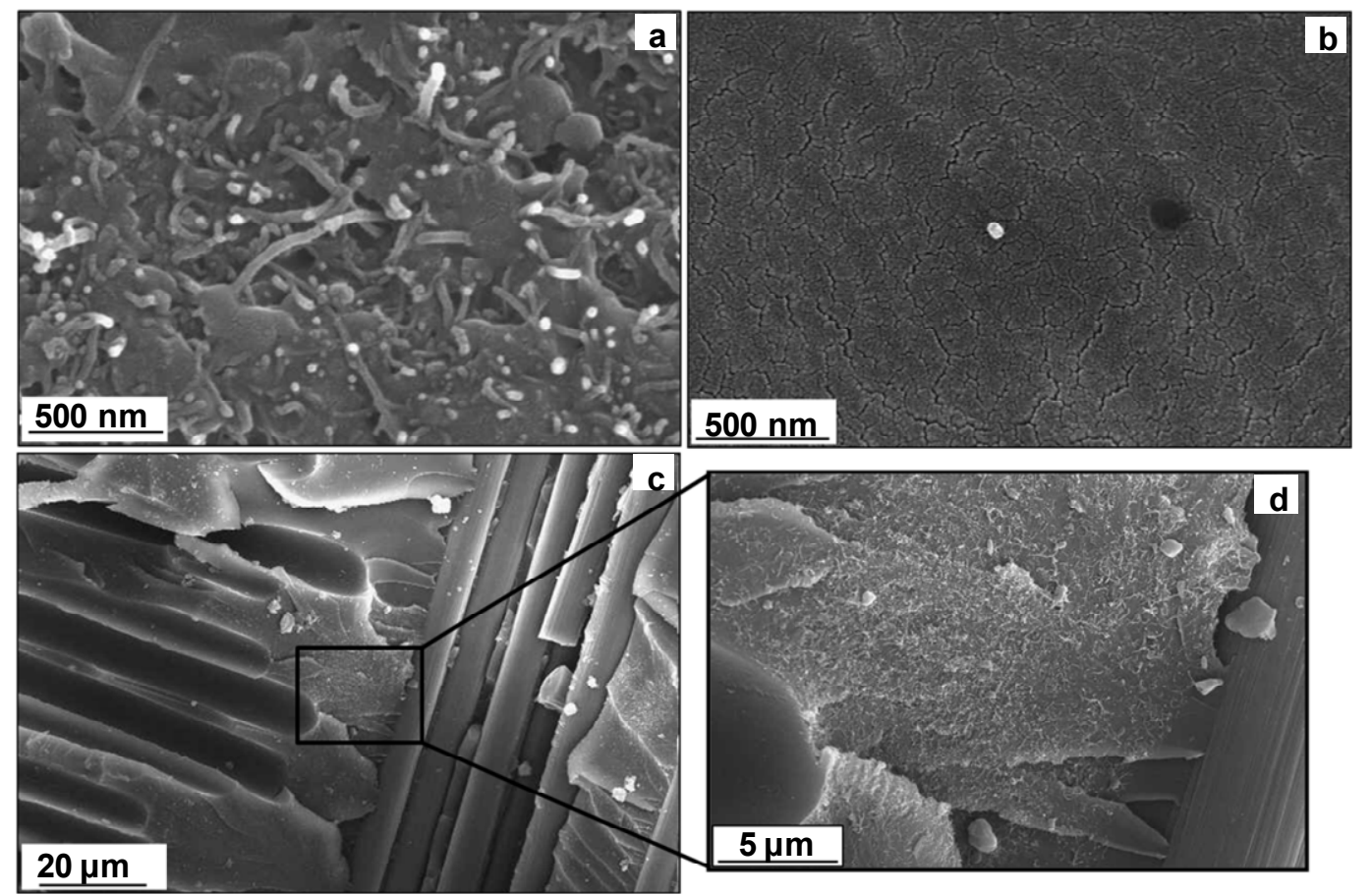

Fig. 13 SEM micrograph of the fracture surface of the P940 filled resin at the inlet (a) and outlet region (b); and delamination surface of the corresponding carbon fibre composite $100 \mathrm{~mm}$ away from the inlet (c) showing CNT filtration at resin rich pockets (d).
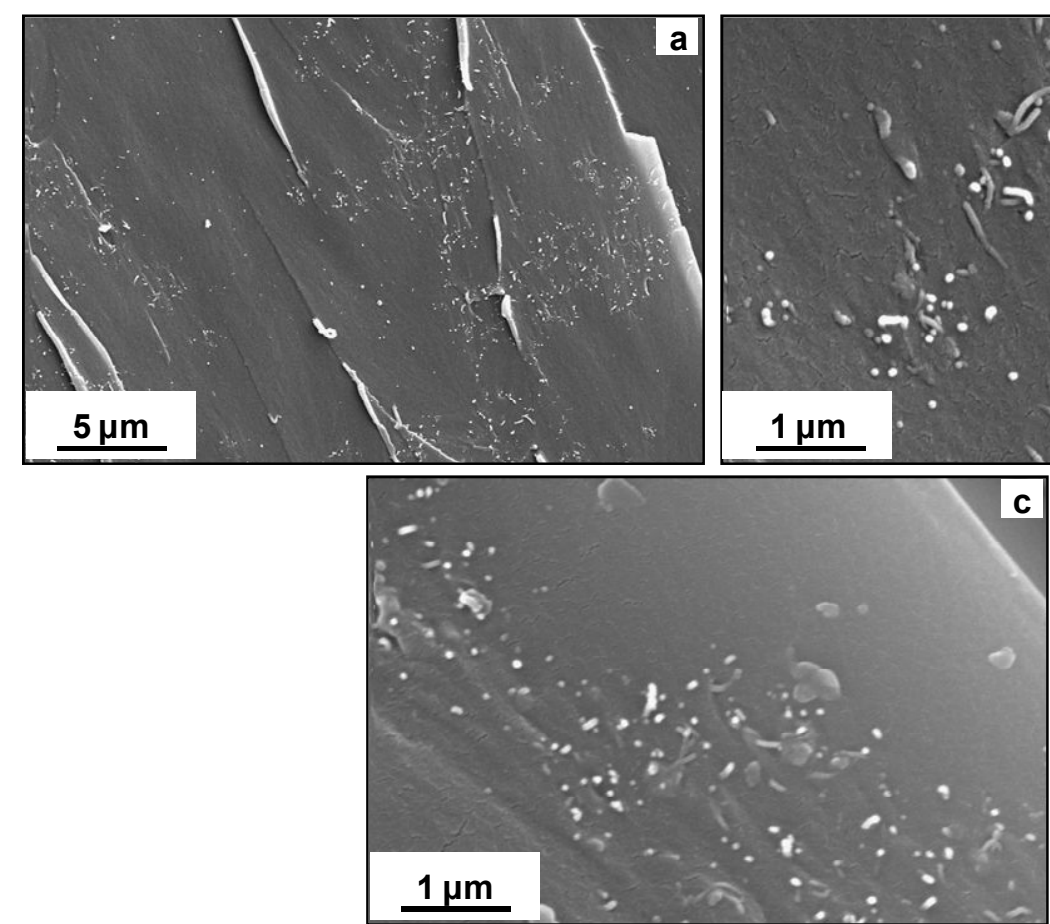

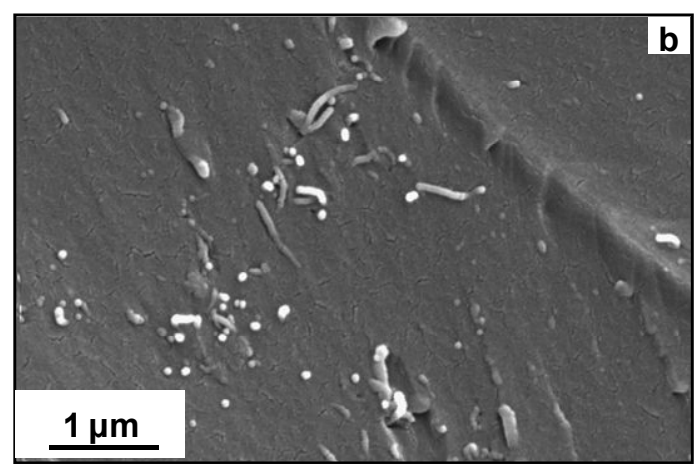

c 
Fig. 14 SEM images of the inlet region ( $a$ and $b$ ) of the $\mathrm{C} 100$ filled resin; and delamination surface of the corresponding glass fibre composite at approximately $130 \mathrm{~mm}$ away from the inlet (c)

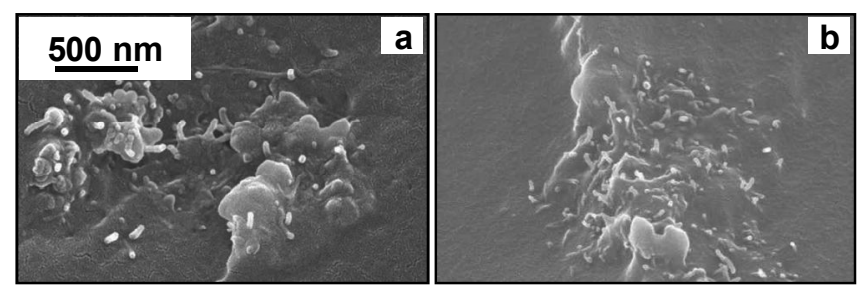

Fig. 15 SEM micrograph of the fracture surface of the carbon fibre composite with surface modified CNTs: inlet (a) and outlet (b) resin. 
a

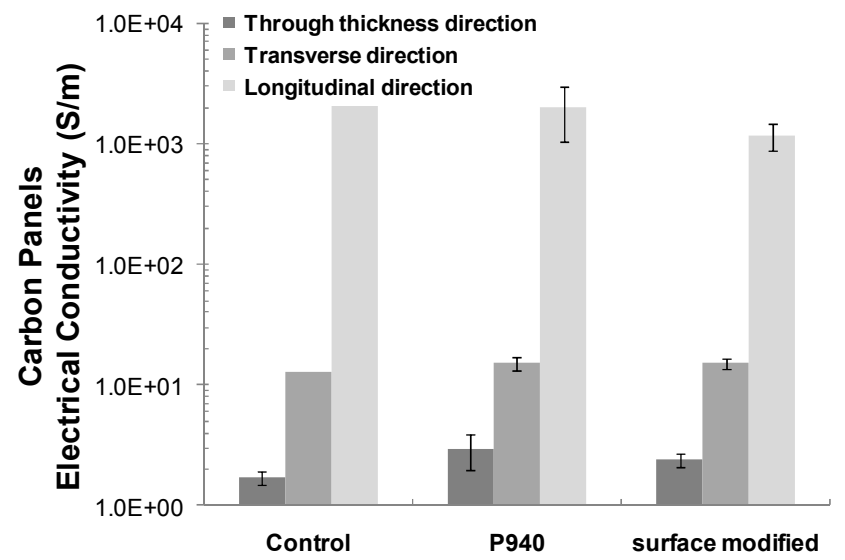

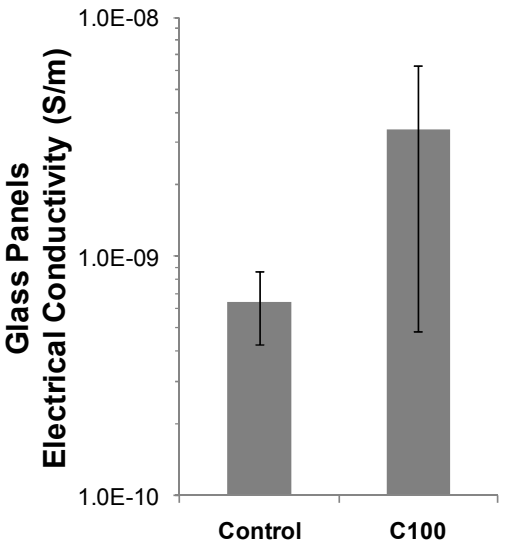

C

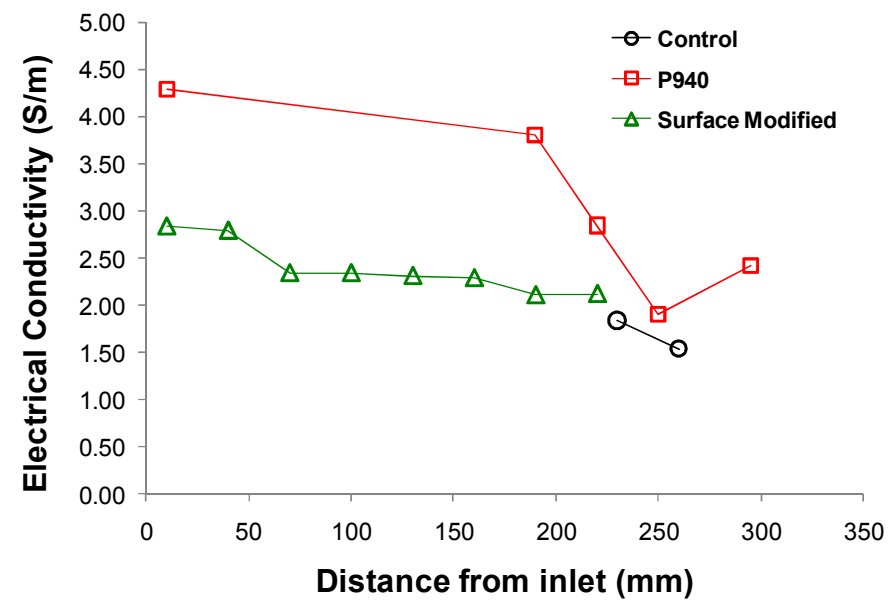

Fig. 16 Electrical conductivity of the CNT filled carbon (a) and glass fibre composites (b), and evolution of through thickness electrical conductivity of the nanoparticle filled carbon composites as a function of the distance from the inlet gate (c). 